\title{
Optimizing the Dispersion Conditions of SWCNTs in Aqueous Solution of Surfactants and Organic Solvents
}

\author{
Md. Mahfuzur Rahman, Hammad Younes, Navaladian Subramanian, and Amal Al Ghaferi \\ Institute Centre for Energy (iEnergy), Mechanical and Materials Engineering Department, \\ Masdar Institute of Science and Technology (MIST), P.O. Box 54224, Abu Dhabi, UAE
}

Correspondence should be addressed to Md. Mahfuzur Rahman; mrahman@masdar.ac.ae

Received 26 June 2014; Accepted 9 September 2014; Published 13 October 2014

Academic Editor: Lian Gao

Copyright (C) 2014 Md. Mahfuzur Rahman et al. This is an open access article distributed under the Creative Commons Attribution License, which permits unrestricted use, distribution, and reproduction in any medium, provided the original work is properly cited.

\begin{abstract}
The optimum concentration of surfactants in aqueous medium and the optimum sonication time for organic medium to achieve the best dispersion of SWCNTs are investigated in this study. Scanning electron microscope (SEM), atomic force microscope (AFM), and UV-vis-NIR study show that the optimum surfactant concentrations to achieve best dispersion for $0.25 \mathrm{mg} / \mathrm{mL}$ of SWCNTs are $9-10 \mathrm{mg} / \mathrm{mL}$ for (sodium dodecylbenzenesulfonate (SDBS)) and 8-9 mg/mL for (sodium deoxycholate (DOC)). The diameter of the SWCNT lies between 0.7 and $2.5 \mathrm{~nm}$ in the case of aqueous solvent, which is in good agreement with the chirality. Moreover, SEM analysis reveals the presence of well-dispersed nanotubes along with some amount of surfactant particles; that is, some nanotubes might be covered with surfactants. Best dispersion of SWCNTs in organic solvents (1,2-Dichloroethane (DCE) and N,N-Dimethylformamide (DMF)) is achieved by four hours of tip sonication. It realized that organic solvents provide pure SWCNTs after drying, which may possibly have SWCNTs with intrinsic properties.
\end{abstract}

\section{Introduction}

Single-walled carbon nanotubes (SWCNTs) are promising materials for many applications such as photovoltaic, medical diagnostics, drug delivery, nanofluids, grease, optical sensing, nanoelectronics, and polymer nanocomposites [1-6]. However, there are a number of challenges for bulk applications that include (1) purification of the nanotubes after synthesis and (2) well dispersing these tubes in solvents or matrix, preferably as individual or small bundles of SWCNTs. Dispersion of carbon nanotubes in water or organic solvents can make them fulfill the requirements for many applications especially in electronic and photonic devices as it is possible to have individual nanotubes with intrinsic properties [7]. Organic solar cells made of single polymer and two electrodes are inefficient because of the low carrier mobility which can be solved by introducing carbon nanotubes (CNTs) $[8,9]$.

SWCNTs could be metallic or semiconducting and act as electron donor or acceptor. Using SWCNTs as donor in the semiconducting polymer matrix improves solar cell performance by providing ballistic pathways for the hole transport, while using SWCNTs as acceptor in organic solar cell has not yet been reported as highly efficient [10]. To explore the unique properties of CNTs, obtaining individual nanotubes are key requirement [11, 12]. However, assynthesized SWCNTs are bundled because of the strong Van der Waals forces. Hence, achieving well-dispersed suspension of SWCNTs without physical or chemical modification of pristine SWCNTs is challenging, but important for various applications.

Significant progress has been achieved in the field of CNTs dispersion in aqueous and organic solvents. A number of research groups have reported various approaches including covalent and noncovalent approaches to obtain stable and homogeneous dispersions of nanotubes [13-21]. Covalent approach requires chemical modification, which causes defects in the nanotube walls and may result in the deterioration of their electrical and mechanical properties [18]. Noncovalent stabilization of SWCNTs has advantage as it preserves the structure and properties of nanotubes $[13,18]$. 
Nowadays, surfactants, polymers, and organic solvents are widely used for the noncovalent stabilization of nanotubes.

Surfactant has hydrophilic and hydrophobic heads and the interactions between them are believed to cause the dispersion of nanotubes in water. The physical mechanism behind the surfactant aided dispersion is that the surfactant adsorbs on the CNT surface by hydrophobic or $\pi-\pi$ interactions and forms a complete or nearly a complete layer that stabilizes separated CNTs from reaggregation. Ultrasonication of the CNT solution with surfactant provides enough energy to separate the nanotubes by overcoming Van der Waals force [13]. During this process, surfactant molecules are adsorbed onto the nanotube surfaces and introduce electrostatic and/or steric repulsion forces that overcome the Van der Waals attraction forces among nanotubes and exfoliate them into individual nanotubes without disrupting the native electronic structure of the nanotubes [18, 21-23]. One of the important dispersion parameters is surfactant concentration, which can affect dispersion. At very low surfactant concentration, the dispersion quality is poor because nanotubes form bundles. At very high surfactant concentration, dispersion quality becomes poor, as the surfactant does not dissolve completely. Thus, there will always be an optimum surfactant concentration for a specific type of SWCNTs at a specific concentration $[15,20]$. The quality of the surfactant is determined by (1) the density of surfactant packing and (2) the ease with which the surfactant can be removed from the surface. Various anionic, cationic, and nonionic surfactants have been used to disperse SWCNTs in aqueous solution effectively [15-17]. However, one has to know the optimum surfactant concentration and the extent to which the suspended material exists as single or small bundles, that is, the extractability of the surfactants.

Short individual SWCNTs with minimum defects are critical to improve solar cell performance. Long nanotubes can develop short circuit by connecting top and bottom layer. Improper handling of SWCNTs can degrade solar cell performance as the incorporated metallic SWCNTs can provide efficient sites for exciton recombination. Moreover, inappropriate energy levels in some semiconducting SWCNTs and poly 3-hexylthiophene (P3HT) can cause energy transfer instead of charge separation and the high length of SWCNTs can result in serious problem of short-circuit in devices [23]. Thus, the dispersion of SWCNTs at optimum condition is important for solar cell application. Dispersion of SWCNTs in organic solvent is physical rather than chemical process as the tubes are not covalently functionalized, that is, without the addition of any other functional group [24]. A number of organic solvents have been reported for the best dispersion of SWCNTs, like DMF (N,N-dimethylformamide), DCE (1,2-dichloroethane), DCB (dichlorobenzene), NMP (N-methylpyrrolidone), chloroform, toluene, epoxy, and so forth [25-30].

Surfactant-aided aqueous dispersion of SWCNTs can individualize nanotubes, but the removal of surfactant is difficult, because of its high boiling point. Surfactants wrap SWCNTs and this may degrade hole transport properties of nanotubes in the organic solar cell. On the other hand, organic solvents can dissolve SWCNTs at very low concentration without altering the properties of pristine nanotubes.
Organic solvents are easy to be removed due to its low boiling point.

In this work, the optimum surfactant's concentration of SDBS and DOC has been determined experimentally by analyzing the semiconducting peak of the UV-vis-NIR absorption spectra where that resonant ratio (peak height to peak width) was calculated and plotted against the surfactant concentration. Wrapping of SWCNTs by surfactant was verified experimentally by EFM (Electrostatic Force Microscopy) analysis. For the best dispersion, the maximum resonant ratio and corresponding surfactant concentration was taken. The optimum sonication time was also determined for the best dispersion of SWCNTs in DMF and DCE. For organic solvents, the effect of centrifugation was characterized through SEM (Scanning Electron Microscope). Finally, a comparative analysis has been made between organic solvent and surfactant aided aqueous dispersion of SWCNTs for photovoltaic application.

\section{Experimental Details}

Materials. SWCNTs with the chirality $(7,6)$, carbon $>90 \%$, $\geq 77 \%$ (carbon as SWCNT), 0.7-1.1 nm diameter, and length, 300-2300 $\mathrm{nm}$ and produced by CoMoCAT catalytic chemical vapor deposition (CVD) method were received from SigmaAldrich. The surfactants, sodium dodecylbenzenesulfonate (SDBS) $\left(\mathrm{C}_{18} \mathrm{H}_{29} \mathrm{NaO}_{3} \mathrm{~S}\right.$; MW $\left.348 \mathrm{~g} / \mathrm{mol}\right)$ and sodium deoxycholate (DOC $\geq 97 \%)\left(\mathrm{C}_{24} \mathrm{H}_{39} \mathrm{NaO}_{4}\right.$, MW $\left.414.55 \mathrm{~g} / \mathrm{mol}\right)$, and the organic solvents, 1,2-Dichloroethane (DCE), anhydrous, 99.8\%; N,N-Dimethylformamide (DMF), anhydrous, $99.8 \%$ used for the dispersion were obtained from Sigma-Aldrich. The manufacturer provides all the details presented here. To prepare the surfactant solutions for the experiments, deionized MilliQ water with a resistivity of $18.2 \mathrm{M} \Omega-\mathrm{cm}$ was used. For the dispersion experiments, $0.25 \mathrm{mg} / \mathrm{mL}$ and $0.05 \mathrm{mg} / \mathrm{mL}$ of the SWCNTs concentration were used for surfactants and organic solvents, respectively.

Instrumentation. Sonication was done using a UP400S $(400 \mathrm{~W}, 24 \mathrm{kHz})$ sonicator with ultrasonic processor, Hielscher, with sonotrode made of titanium and tip diameter $3 \mathrm{~mm}$, approximately $100 \mathrm{~mm}$ length was used. To control the temperature, a large water bath was used during sonication. The frequency was fixed at $100 \%$ and the amplitude was always kept at $30 \%$ for surfactants and at $50 \%$ for organic solvents. Tip sonication provides high energy that can exfoliate nanotube bundles and shortens tube length, which are favorable for photovoltaic application [22, 23, 25]. It is well known that each solvent has its solvation capacity for dissolving nanotubes and sonication time does not alter this capacity. However, solvents can dissolve SWCNTs with reduced bundle size with the increased duration of sonication and energy [20, 22]. Experiments were performed by varying sonication time to find an optimum time to achieve better dispersion.

UV-vis-NIR spectrophotometer (PerkinElmer LAMBDA 1050) was used to study the optical properties of the SWCNTs solutions. It was operated between $300 \mathrm{~nm}$ and $1400 \mathrm{~nm}$ with $3 \mathrm{~nm}$ increment with the use of $10 \mathrm{~mm}$ path length quartz cuvettes and a surfactant solution for the baseline correction. 
To study the morphology of the SWCNTs, atomic force microscopy (Asylum MFP-3D, AFM) and scanning electron microscope (FEI quanta 250 ESEM) were used. The diameter of SWCNTs was calculated from the AFM image. It can be concluded from the micrograph that the dispersion is better if the bundle diameter is very similar to the individual tube diameter.

Procedures. For each experiment, $0.25 \mathrm{mg} / \mathrm{mL}$ SWCNTs was mixed with $15 \mathrm{~mL}$ of aqueous solution of a surfactant containing different concentrations, ranging from 3 to $20 \mathrm{mg} / \mathrm{mL}$, of sodium dodecylbenzesulfonate (SDBS) and sodium deoxycholate (DOC). Firstly, the surfactant solution was prepared by maintaining the foam formation at minimum level because the formation of foam affects the dispersion. Then, the resulting mixture of SWCNTs was ultrasonicated for 2 hours as reported as optimum by Tan and Resasco [13]. After completion of the sonication process, the sample was diluted by 10 times to record the UV-vis-NIR absorption spectra.

In case of organic solvents, $0.05 \mathrm{mg} / \mathrm{mL}$ of SWCNTs was mixed with $25 \mathrm{~mL}$ of a solvent for each experiment. Resulting mixture of SWCNTs was ultrasonicated for 1, 2, 3 , and $4 \mathrm{~h}$. Figure 1(a) shows the dispersion state of samples immediately after ultrasonication. After completion of the sonication process, the mixture was aged for $2 \mathrm{~h}$ for the sedimentation of undissolved or undispersed nanotubes so that those particles would not interfere during the UV-visNIR measurement. Figure 1(b) shows the sedimentation of the undissolved nanotubes where the transparent part of the solution is dissolved nanotubes in the organic solvent that was used for the characterization. Then, a glass pipette was used to transfer the solution containing dissolved nanotubes to record the UV-vis-NIR absorption spectra. For AFM and SEM studies, dip coating was used to prepare samples on silicon $(\mathrm{Si})$ substrates. The substrates were cleaned by bath sonication in ethanol for $30 \mathrm{~min}$ followed by washing with acetone. Furthermore, the effect of centrifugation was studied by SEM. A constant volume of the solution was centrifuged for $15 \mathrm{~min}$ at $6000 \mathrm{rpm}$ and the resultant solution was used to prepare SEM sample. In addition, the centrifuged solution was resonicated for $15 \mathrm{~min}$ and characterized by SEM.

\section{Results and Discussion}

The effective dispersion of the suspension can be judged by visual inspection as well-dispersed suspension shows no precipitation and no visible small particles. In general, if centrifugation is used after sonication it results in black and more homogeneous suspensions. In the absence of centrifugation of the aqueous SWCNTs suspension, the optical absorption spectra show large background absorption. Naumov et al. have recently showed that the background could be induced by ultrasonication of SWCNTs and surfactants and the differences in the background absorption are related to the nanotube lengths, plasmonic absorption of SWCNTs and carbonaceous impurities, contributions from chemical derivations, spectral broadening from nanotube bundling, and spectral congestion from closely spaced peaks in samples with many $(n, m)$ species [31]. However, only ultrasonication was used for the dispersion before characterization. Sonication process is the most crucial step in the dispersion process and the most of nanotubes debundle during this process.

The effect of sonication on surfactant was examined using SEM. The sonication applies harsh condition and induces significant systematic increases in background UVvis absorption. The prolonged tip sonication of surfactant solution can cause the formation of small particles that are significantly denser than water, presumably through chemical reactions of surfactant during solvent cavitation (Figure 2). The morphology of the particles, as can be seen in Figure 2, was ellipsoidal (mostly non-spherical) and their average length was in the range of 2.5 to $12 \mu \mathrm{m}$. Thus, the optical study of dispersion without centrifugation can provide information about the nanotube length, percentage of metallic tubes, aggregated nanotubes, and the effect of sonication and surfactant.

3.1. Dispersing Ability of SDBS and DOC Using UVvis-NIR Spectroscopy. A constant SWCNTs concentration $(0.25 \mathrm{mg} / \mathrm{mL})$ with different surfactant concentration was used to determine the optimum SWCNTs to surfactant ratio for better dispersion in aqueous medium. The UVvis-NIR study of the SWCNTs solutions is shown in Figure S2 (Supporting Information, available online at http://dx.doi.org/10.1155/2014/102621) and the absorption at wavelength $850 \mathrm{~nm}$ where no characteristic peaks observed were used for calculation. SWCNTs concentration after dispersion was calculated using Beer-Lambert law. SWCNTs concentration in the solution after dispersion was compared with the initial concentration of the nanotubes and this was done by calculating the percentage of SWCNTs after dispersion, which was termed as extractability. The results are shown in Figure 3 where the extractability of each surfactant (from equation (b), Supporting Information) has been plotted as a function of the surfactant concentration.

Figure 3(a) shows the variation in extractability for different SDBS surfactant concentrations and Figure 3(b) is the variation in extractability for different DOC surfactant concentrations. For SDBS, the surfactant concentrations 0.03 , $0.04,0.05,0.06,0.07,0.08,0.09,0.1,0.11,0.12,0.14,0.16$, $0.18,0.2,0.25$, and $0.3 \% \mathrm{~W} / \mathrm{V}$ and, for DOC, the surfactant concentrations $0.03,0.05,0.07,0.09,0.1,0.11,0.13$, and $0.15 \% \mathrm{~W} / \mathrm{V}$ were used and we found that there exist a range of optimum concentrations for which the exfoliation of nanotube bundles is maximum at the specific dispersion condition. Here, quadratic correlation was used between percentage extractability and the concentration of surfactant. From Figure 3(a), it is obvious that about 95\% exfoliation is possible at the 10 to $13 \mathrm{mg} / \mathrm{mL}$ SDBS concentrations; that is, in the final solution the size of the nanotubes are very small, similarly, from Figure 3(b), the optimum DOC concentration was found to be about 8 to $10 \mathrm{mg} / \mathrm{mL}$ and the maximum possible exfoliation is about $70 \%$, which means that some nanotubes still exist in bundles, which are bigger enough to precipitate. Thus, the better dispersion of SWCNTs can be obtained by using 10 to $13 \mathrm{mg} / \mathrm{mL}$ ( 0.1 to $0.13 \% \mathrm{~W} / \mathrm{V}$ ) of SDBS and 8 to $10 \mathrm{mg} / \mathrm{mL}(0.08$ to $0.1 \% \mathrm{~W} / \mathrm{V})$ of DOC 


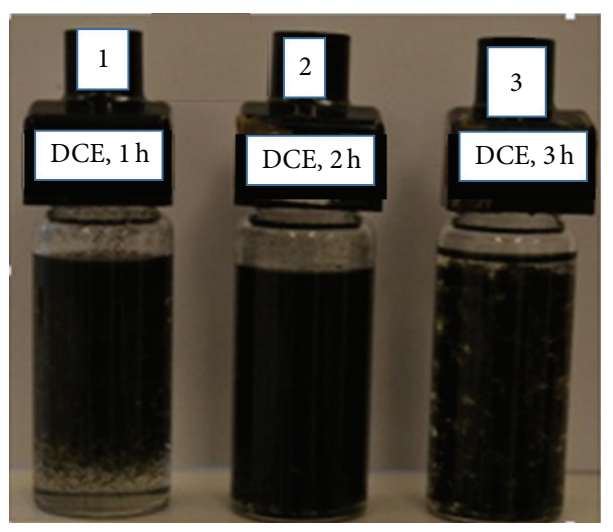

(a)

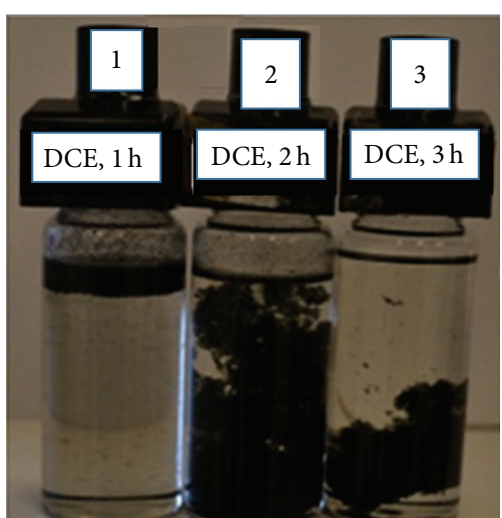

(b)

FIGURE 1: Photographs of SWCNTs in DCE with different sonication times, sample 1 for one hour, sample 2 for two hours, and sample 3 for three hours. Photograph (a) shows the state of the SWCNTs mixture samples 1, 2, and 3 immediately after sonication and photograph (b) shows the state of the SWCNTs mixture samples 1,2 , and 3 after $2 \mathrm{~h}$.

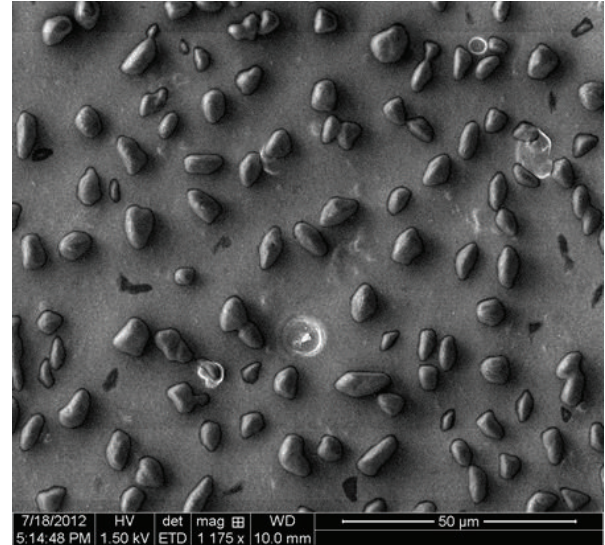

FIGURE 2: SEM image of the tip-sonicated SDBS solution after drying. It shows the formation of small particles that might cause background absorption in UV-vis-NIR absorption spectra.

surfactant concentration for $0.25 \mathrm{mg} / \mathrm{mL}$ SWCNTs that was tip-sonicated for $2 \mathrm{~h}$.

The extractability analysis reveals that the dispersion ability of SDBS is better than the dispersion ability of DOC. The chemical structure of SDBS has a benzene ring and the presence of the benzene ring in the surfactant structure increases the dispersion power of a surfactant because of $\pi-\pi$ interactions. Surfactants are believed to work as better dispersant above the critical micelle concentration, and there always exists an optimum surfactant concentration for which nanotube exfoliation is high [13]. With the increase of surfactant concentration, size of micelle increases so that it could form multilayers of surfactant on the nanotube surface. Thus, the bridging of SWCNTs via surfactant could occur due to the interaction of surfactant molecules at very high concentration and this might cause nanotubes flocculation. This flocculation leads to a decrease on the dispersion of nanotubes. From the analysis, it was also observed that at very high surfactant concentration the extractability decreases.
3.2. Determination of the Optimum Surfactant Concentration Using Near IR Absorption Spectra. Well-resolved optical absorption spectra can be used to measure the level of dispersion as well as the determination of the extractability of each surfactant. The characteristic optical features, that is, the absorption peaks, can be seen clearly by the wellresolved optical absorption spectra. It is widely believed that well dispersed and individual nanotubes create narrow and sharp absorption peaks whereas aggregation of nanotubes broadens the absorption peaks [13]. The resolution of these optical absorption spectra can be analyzed by calculating the ratio of peak height to the width of a peak, or the resonant ratio, that is, the ratio of the resonant band area to noresonant background. Thus, the ratio of peak height to width was calculated to find out the possible maximum ratio where the surfactant concentration and the exfoliation of SWCNT bundles are optimum. In Figure 4, the ratio of the peak height to width as a function surfactant concentration was plotted and a polynomial fit was used to get the optimum ratio for each surfactant.

As it can be seen in Figure 4(a), the maximum resonant ratio occurs at SDBS concentration of about $10 \mathrm{mg} / \mathrm{mL}$. However, it was found from the extractability that the optimum SDBS concentration was in the range of 10 to $13 \mathrm{mg} / \mathrm{mL}$. Thus, $10 \mathrm{mg} / \mathrm{mL}$ SDBS concentration could be considered as the optimum concentration, because increasing the surfactant concentration does not increase the resonant ratio much. In Figure $4(\mathrm{~b})$, the optimum DOC concentration is $8 \mathrm{mg} / \mathrm{mL}$ corresponding to the maximum resonant ratio. From the comparative analysis of SDBS and DOC, it is obvious that SDBS has better dispersing power for the SWCNTs with the chirality $(7,6)$.

3.3. Determination of Optimum Sonication Time for Organic Solvents Using Near IR Absorption Spectra. Similarly, for organic solvents, the resonance ratio (peak height to peak width) of the semiconducting peak in the wavelength range of 1200 to $1350 \mathrm{~nm}$ was calculated for DCE and DMF for 


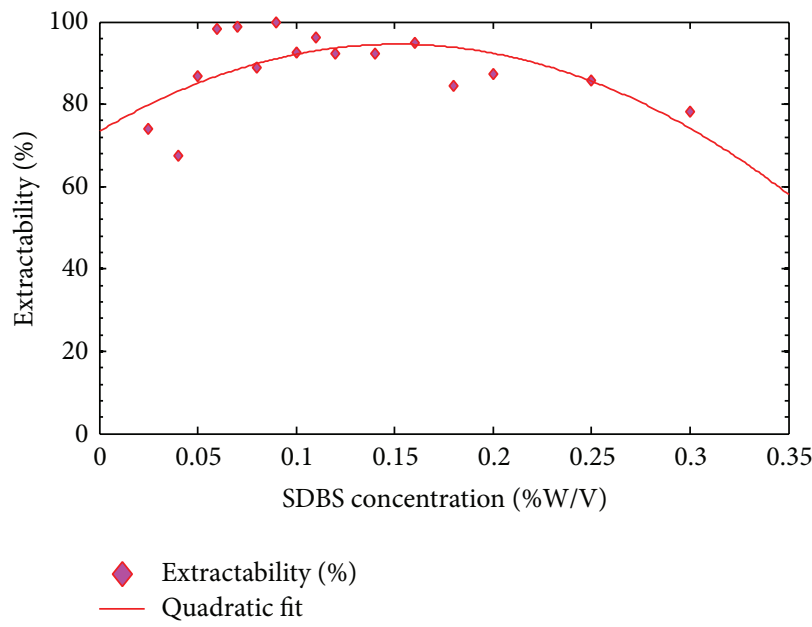

(a)

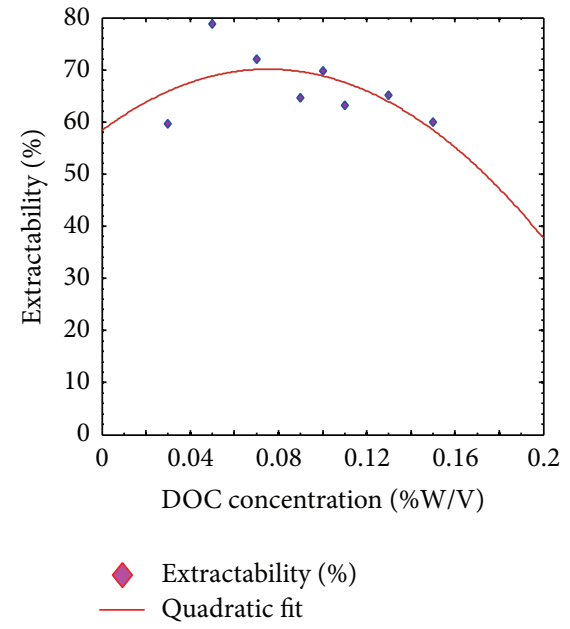

(b)

FIGURE 3: Extractability of (a) SDBS (b) DOC calculated based on the absorption at $850 \mathrm{~nm}$ wavelength at different surfactant concentrations.

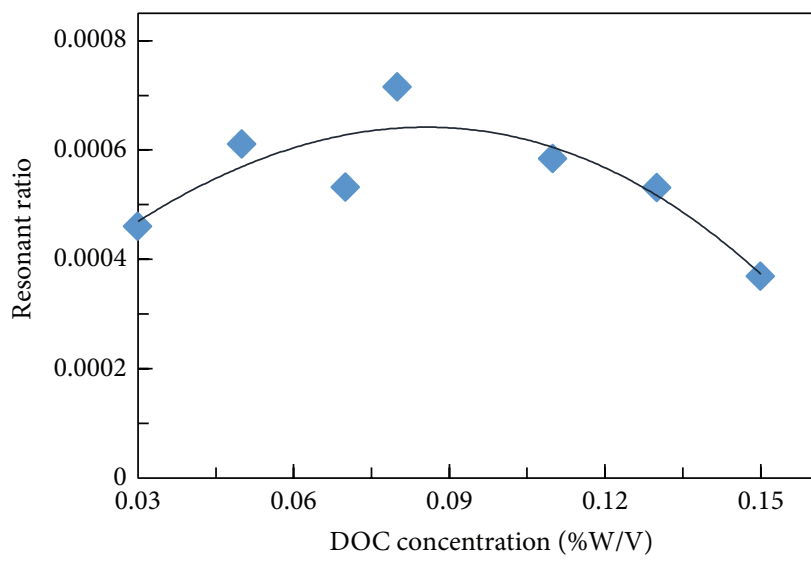

(a)

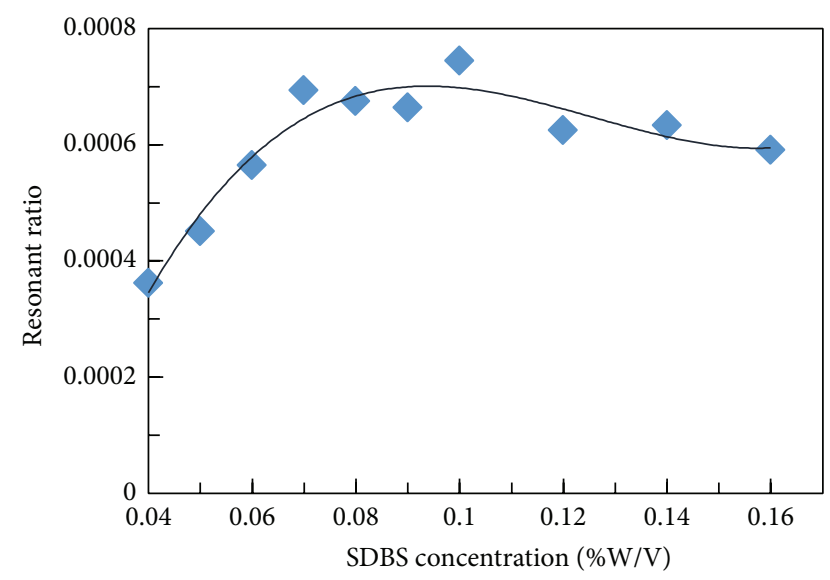

(b)

FIgURE 4: Variation in the resonant ratio with the surfactant concentrations. (a) SDBS surfactant and (b) DOC surfactant.

each dispersion condition. The UV-vis-NIR spectra of the SWCNT solutions of organic solvents are shown in Figure S3 (supporting information). UV-vis-NIR absorption spectra corresponding to $4 \mathrm{~h}$ tip sonication show a peak with higher resolution. The resonant ratio is also maximum for $4 \mathrm{~h}$ tip sonication. This observation implies that the increase in ultrasonication time reduces average bundle size of SWCNTs.

For the DCE solvent, $4 \mathrm{~h}$ of tip sonication at $50 \%$ power output is found to yield a solution with well-dispersed SWCNTs as the resonant ratio is increased considerably as seen in (Figure 5(a)). From Figure 5(b), it is observed that the resonance ratio does not change much with the increase in sonication time. However, longer sonication time is required to reduce the length of nanotube and average bundle size. Therefore, the optimum sonication time for the dispersion was $4 \mathrm{~h}$ tip sonication and the tip sonication provides sufficient energy for nanotubes exfoliation.
3.4. Morphology of SWCNT Dispersion. The morphology study of dispersion using AFM, SEM, and TEM are very essential as they provide better understanding and confidence about the quality of dispersion, although the optical absorption study of dispersion is widely used and gives quite reliable information about dispersion. As complementary techniques to UV-vis-NIR absorption spectroscopy, AFM and SEM were used to characterize dispersion.

Carbon nanotubes (CNTs) show poor solubility in organic solvents. However, such CNTs solutions would be sufficient enough to be usable in solar cells fabrication. Besides, lower amount of CNTs loading has shown better efficiencies than the higher amount. From the SEM image (Figures 6 and 7), it can be seen that nanotubes are mostly individual and they are well dispersed. There are some nanotube bundles but their dimensions seem quite low. To get an idea about the nanotube and bundle diameter, AFM 


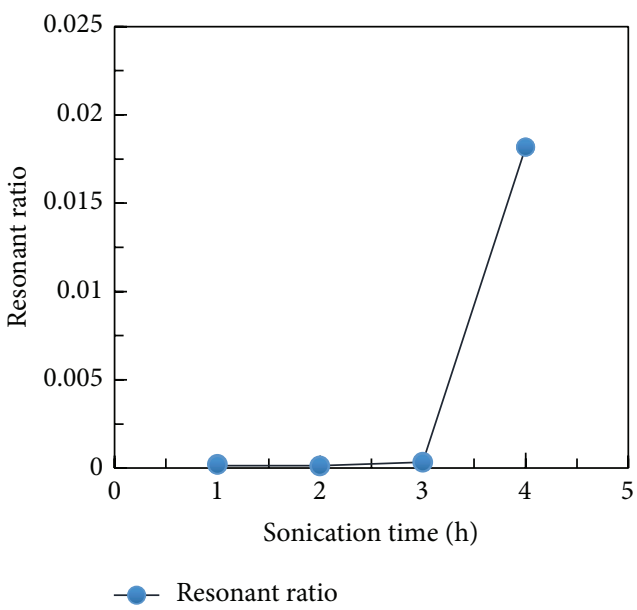

(a)

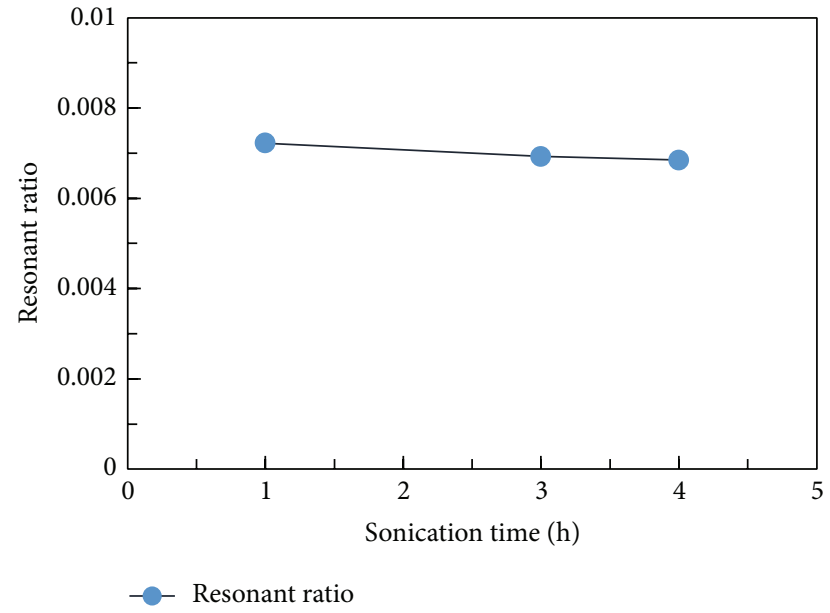

(b)

FIGURE 5: Resonant ratio of the semiconducting peak for different samples of SWCNTs (a) in 1,2-dichloroethane and (b) in N,Ndimethylformamide. This plot shows the resonance ratio of samples with different sonication conditions. Resonance ratio is maximum for the highest debundling of nanotubes.

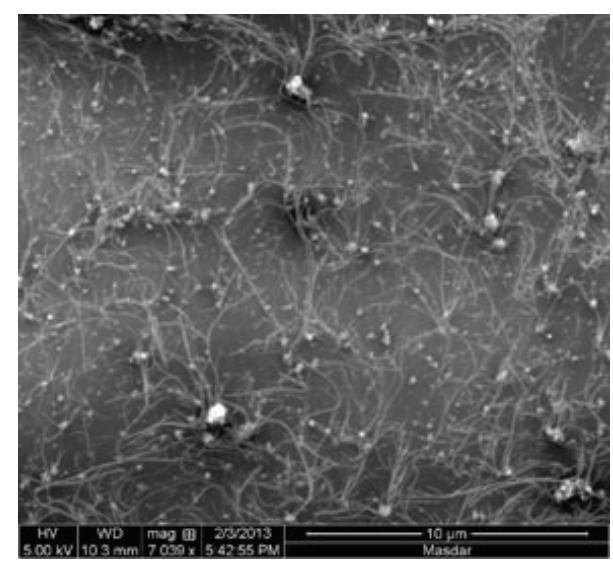

(a)

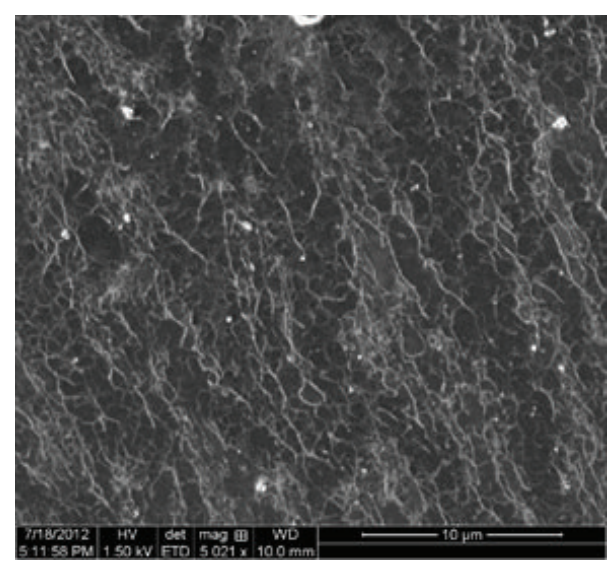

(b)

FIgURE 6: SEM images of the SWCNTs (a) in SDBS ( $8 \mathrm{mg} / \mathrm{mL})$ and (b) in DOC $(10 \mathrm{mg} / \mathrm{mL})$.

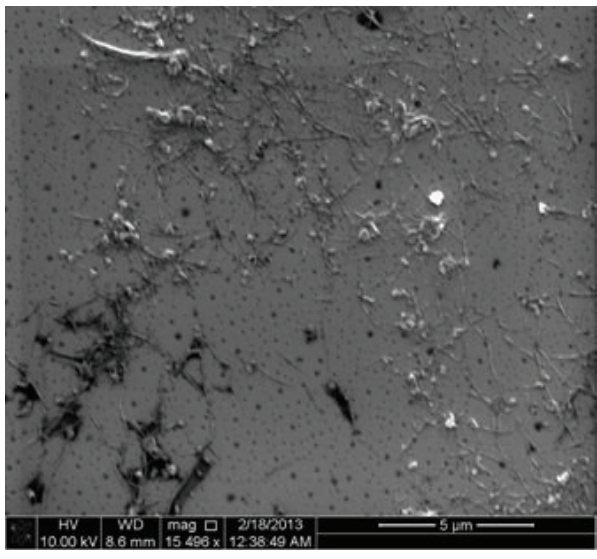

(a)

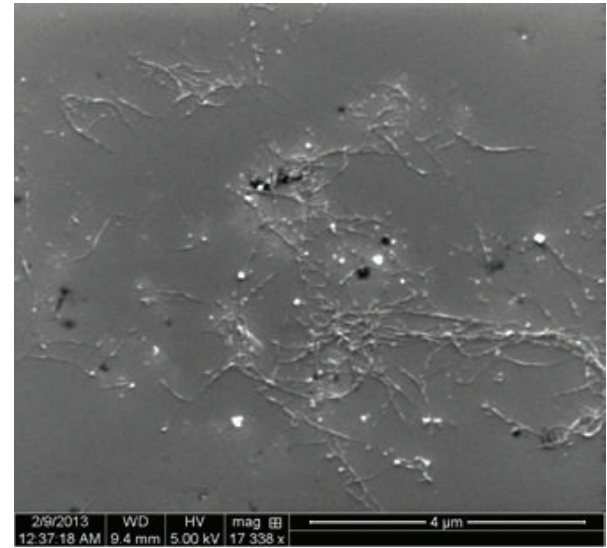

(b)

FIGURE 7: SEM images of SWCNTs coated using (a) DCE-SWCNTs and (b) DMF-SWCNTs solutions. Images show the dispersed state of SWCNTs in DCE and DMF after $4 \mathrm{~h}$ of tip sonication. 


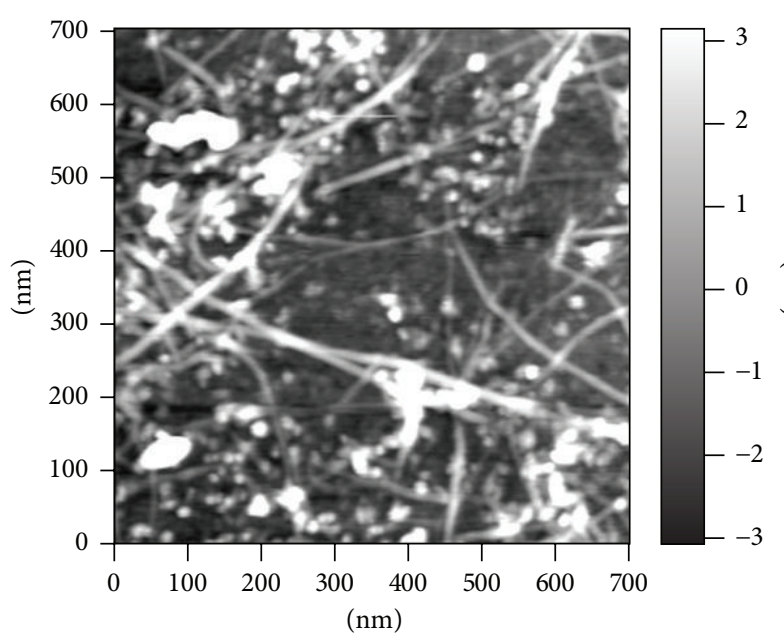

(a)

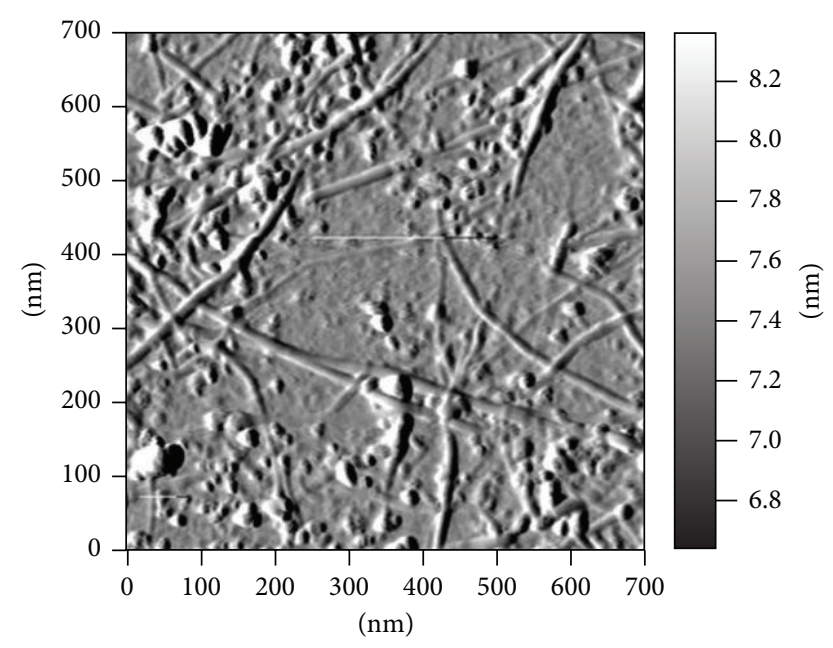

(b)

FIGURE 8: AFM images of SWCNTs with SDBS $(8 \mathrm{mg} / \mathrm{mL})$. The sample was prepared immediately after the dispersion. Image (a) shows the height of the nanotubes. Image (b) shows the phase of nanotubes and impurities present in the sample. SWCNTs are found to be individual after dispersion as almost no entanglement is observed.

sample (Figure 8) was prepared on glass and silicon to get high magnification image of individual SWCNTs.

From Figure 8, it is obvious that the dispersion of SWCNTs is good but there are impurities. These impurities could be surfactants, catalyst particles, or amorphous carbon.

3.5. Diameter Distribution of Nanotubes after Dispersion. From Figure 9, it is obvious that the average bundle size of the nanotubes after dispersion lies in the range of $0.7-2.5 \mathrm{~nm}$. This result indicates that the aqueous solution of surfactant individualizes the nanotube bundles. Herein, the concentrations of SDBS and DOC are optimized by determining the minimum concentration needed to have stable dispersion.

From Figure 10, the average bundle size of SWCNTs is found to be in the range of 1 to $20 \mathrm{~nm}$. These samples were prepared without centrifugation which can remove larger bundles to make a homogenious solution of SWCNTs.

3.6. Effect of Centrifugation on the Dispersion. For many applications, pure and well-dispersed solution of individual SWCNTs is required. Centrifugation is used to remove impurities and bundles of nanotubes. Here, the dispersed solution of SWCNTs in DCE was centrifuged for $15 \mathrm{~min}$ at $6000 \mathrm{rpm}$ and then the SEM analysis of the pure solution was done. From Figure 11(a), it is observed that SWCNTs agglomerate after centrifugation steps and they form Bucky paper of SWCNTs due to strong Van der Waals force. Therefore, the centrifuged solution was resonicated for $15 \mathrm{~min}$.

From Figure 11(b), it is obvious that the resonication of the centrifuged solution plays a crucial role towards obtaining a homogeneous dispersion of SWCNTs with smaller bundles and that the apparent bundle size is in the range of 40 to $50 \mathrm{~nm}$. These bundles size can be reduced by applying sonication for longer periods.
3.7. Comparative Analysis between the Dispersion of Nanotubes in Water and Organic Solvents for Photovoltaic Application. It is obvious that the surfactant-aided dispersion of SWCNTs in water can create a solution of individual nanotubes. From the AFM analysis (Figure 9) it was found that average size of the nanotubes is in the range of 0.7 to $2.5 \mathrm{~nm}$, which confirms the specification provided by the supplier. In contrast, it was found that the average bundle size of the nanotubes in organic solvents lies in a wide range of 1 to $20 \mathrm{~nm}$. However, for photovoltaic application of SWCNTs, organic solvents seem to be preferable as it reduces time and cost and they can be removed easily by evaporation. On the other hand, the removal of surfactant is very difficult due to their high boiling point, and the surfactant wraps the nanotube, possibly disrupting the CNT-polymer contact properties. From the electrostatic force microscopy (EFM) analysis, it is found that surfactants wrap the nanotubes and remain there even after washing with copious amount of DI water (Figure 12). In AFM, the tip-sample interactions depend on the surface properties in the nanoscale. SWCNTs with surfactant will have different interactions than SWCNTs without surfactant. Here, both wrapped and lightly wrapped SWCNTs are present and from the Nap phase (Figure 12(b)); different phase contrast is observed depending on the degree of wrapping.

\section{Conclusions}

In this paper, the dispersion of SWCNTs in different solvents using surfactants has been investigated in order to find the optimum dispersion conditions. Best dispersion of $0.25 \mathrm{mg} / \mathrm{mL}$ of SWCNTs was obtained for $9-10 \mathrm{mg} / \mathrm{mL}$ SDBS surfactant and $8-9 \mathrm{mg} / \mathrm{mL}$ DOC surfactant. Under the same reporting conditions, it was found that the optimum sonication time to obtain good dispersion in organic solvent was about $4 \mathrm{~h}$ where $2 \mathrm{~h}$ of tip sonication was enough for 


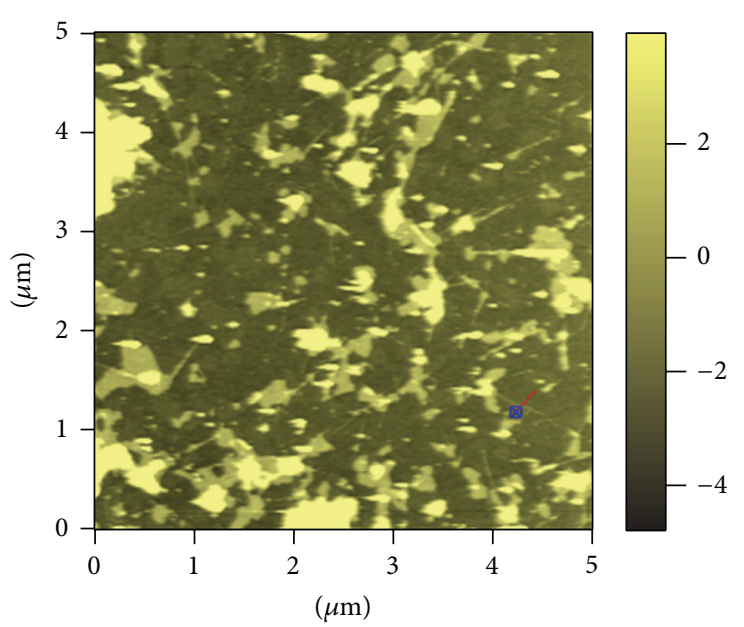

(A)

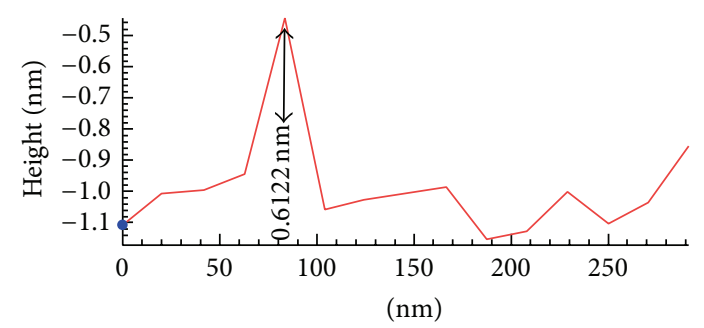

(a)

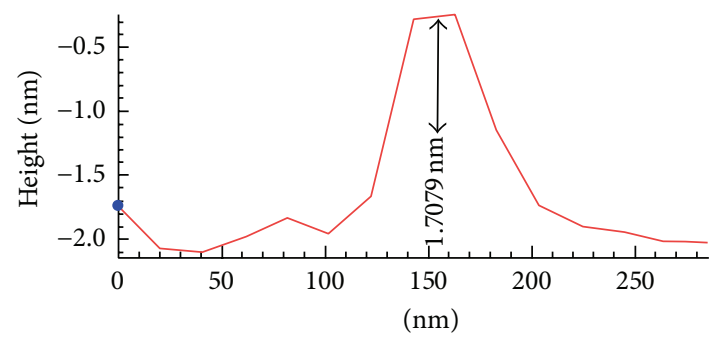

(b)

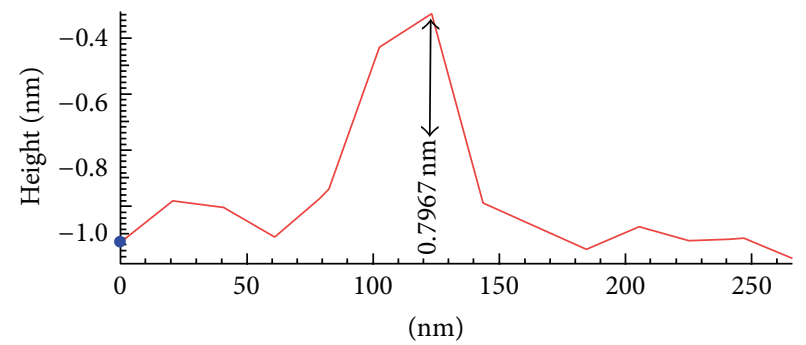

(c)

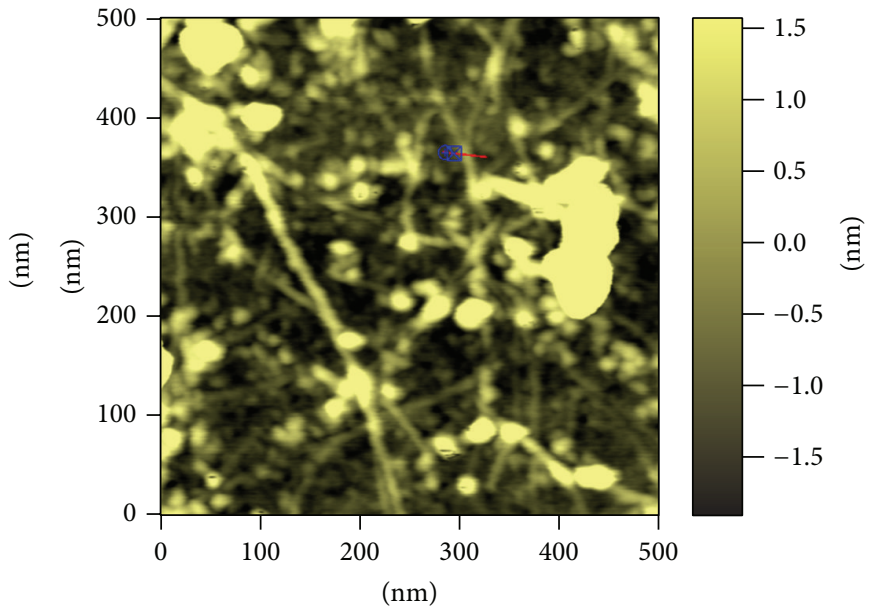

(B)

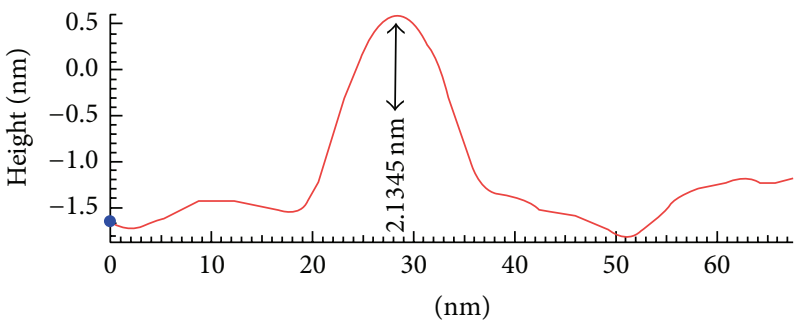

(a)

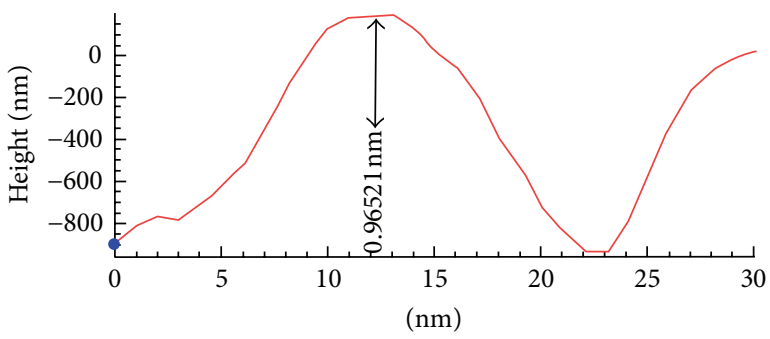

(b)

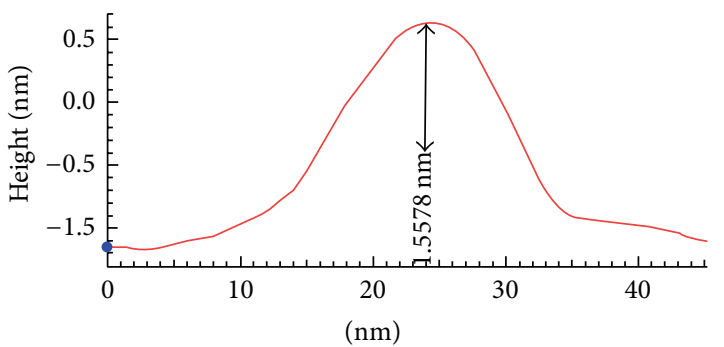

(c)

Figure 9: AFM images and their diameter distributions in (A) DOC (10 mg/mL) and (B) SDBS (8 mg/mL). Average diameter of SWCNTs bundle lies in the range of 0.6 to $2.5 \mathrm{~nm}$.

the surfactant aided dispersion. The diameter distribution of the SWCNTs was found to be between 0.7 and $2.5 \mathrm{~nm}$ in the case of surfactant aided dispersion, which was in good agreement with the chirality. Moreover, SEM analysis revealed the presence of well-dispersed nanotubes along with some amount of surfactant particles, that is, some nanotubes might be covered with surfactants.
The low boiling point of organic solvents provides advantages over surfactant-aided aqueous medium, because it is very easy to remove organic solvents by evaporation from SWCNTs mixture. Although the aqueous solution of surfactants can individualize SWCNTs largely than organic solvent, it is difficult to remove the surfactants that wrap nanotubes and hence the solar cell performance is affected. 


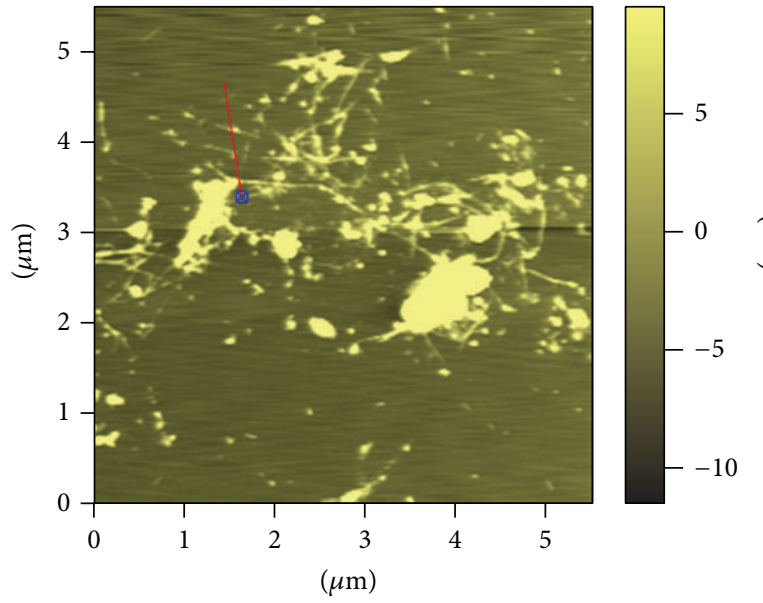

(A)
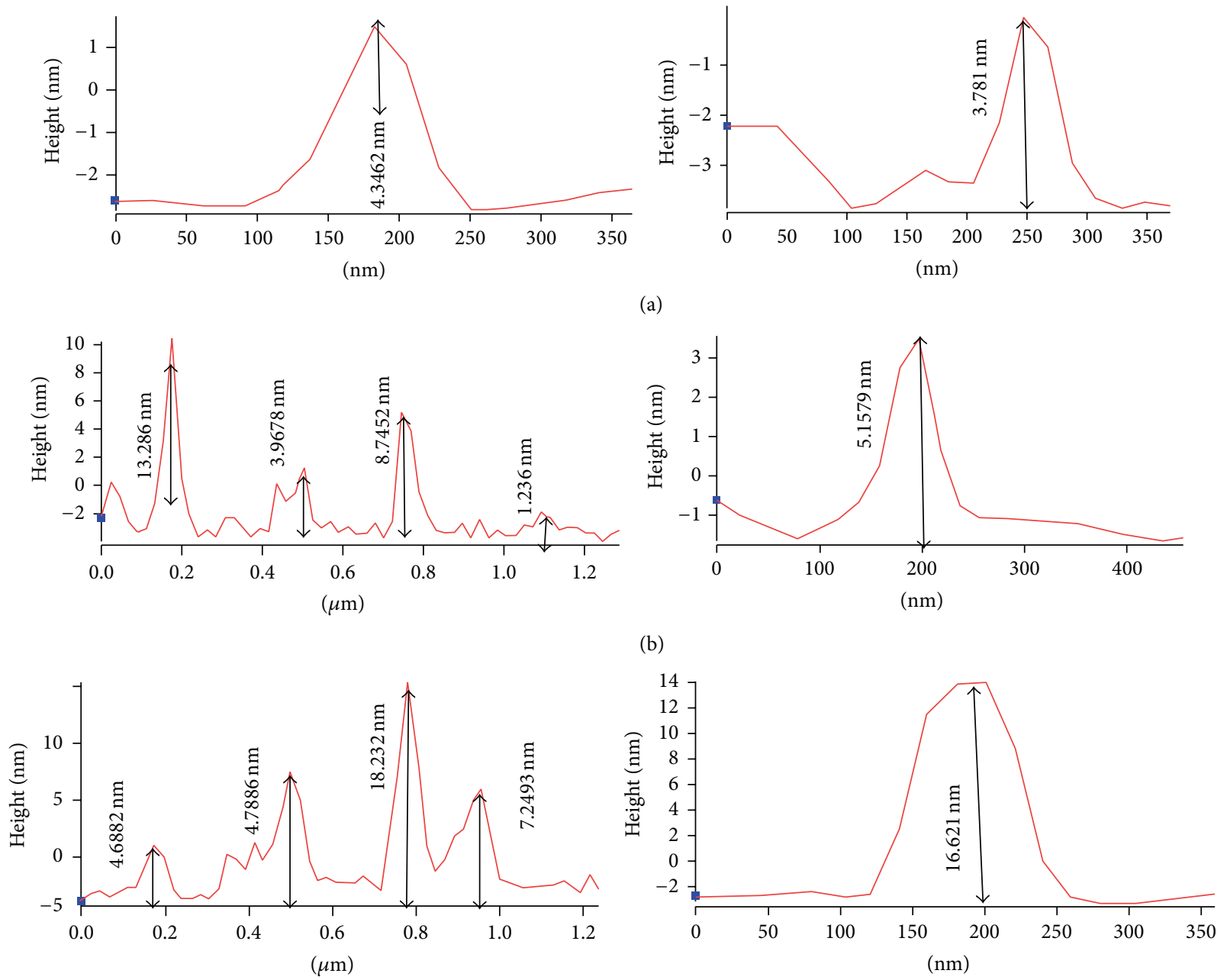

FIGURE 10: AFM analysis of SWCNTs in DMF where (A) and (B) show two different scans. The distribution of bundle sizes lies in the range of 1 to $20 \mathrm{~nm}$. (a)

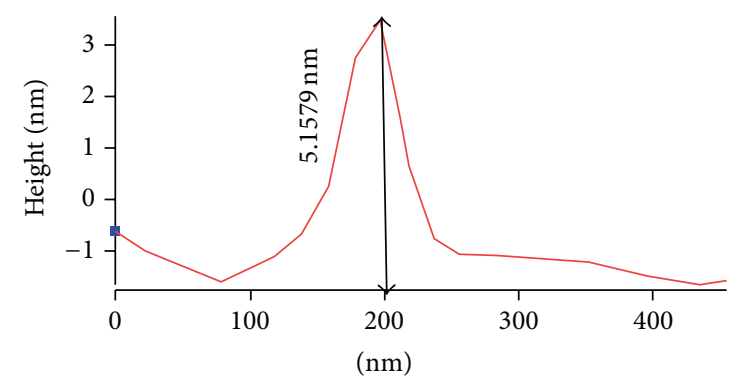

(b)

(c)

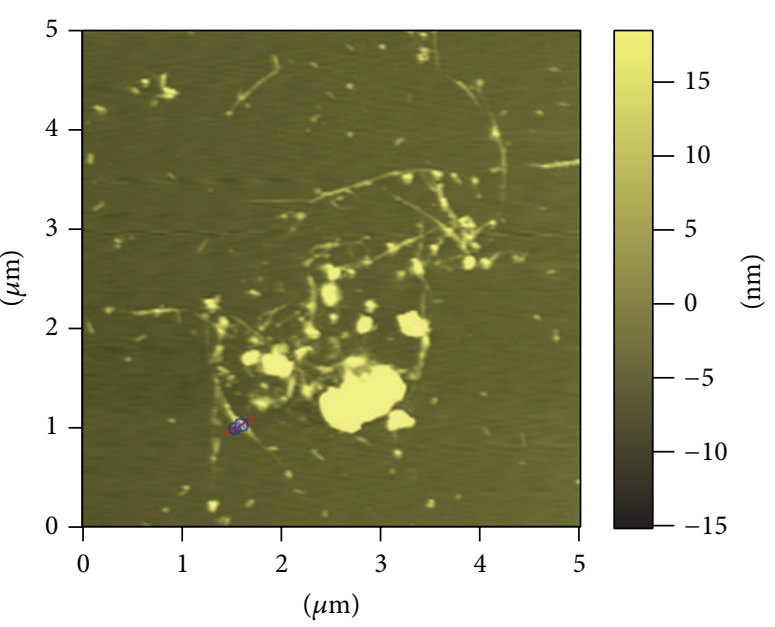

(B)

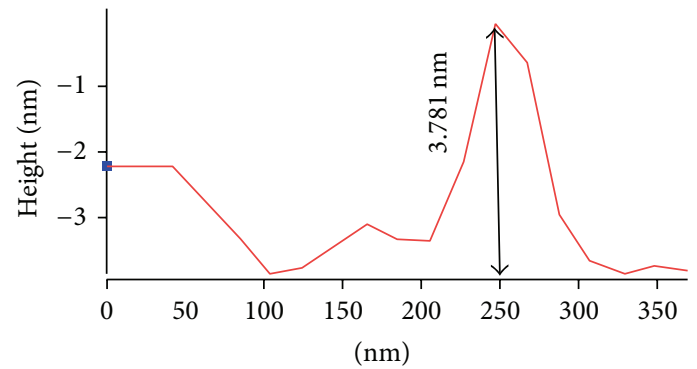




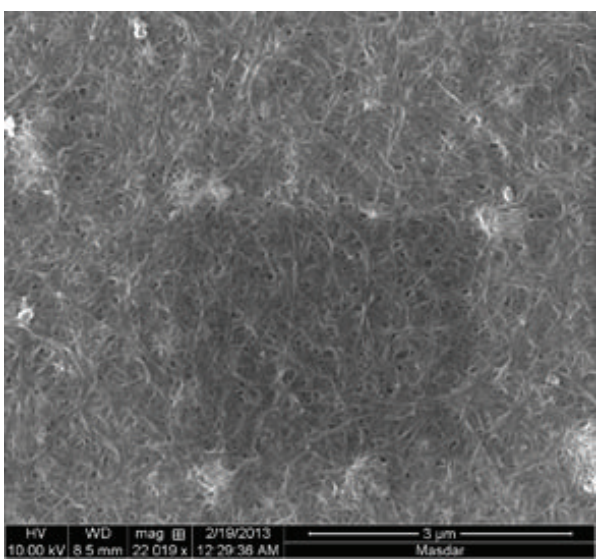

(a)

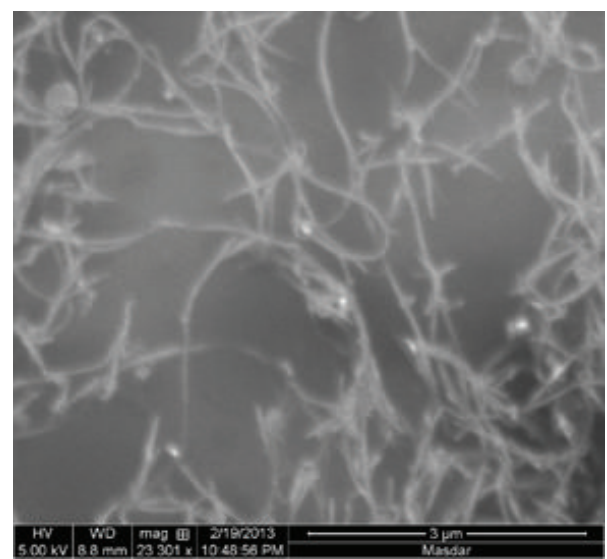

(b)

FIGURE 11: SEM images of SWCNTs. (a) shows agglomeration of SWCNTs formed by $15 \mathrm{~min}$ of centrifugation at $6000 \mathrm{rpm}$ and (b) shows dipersed state of SWCNTs in DEC after 15 min of tip resonication at 50\% amplitude.

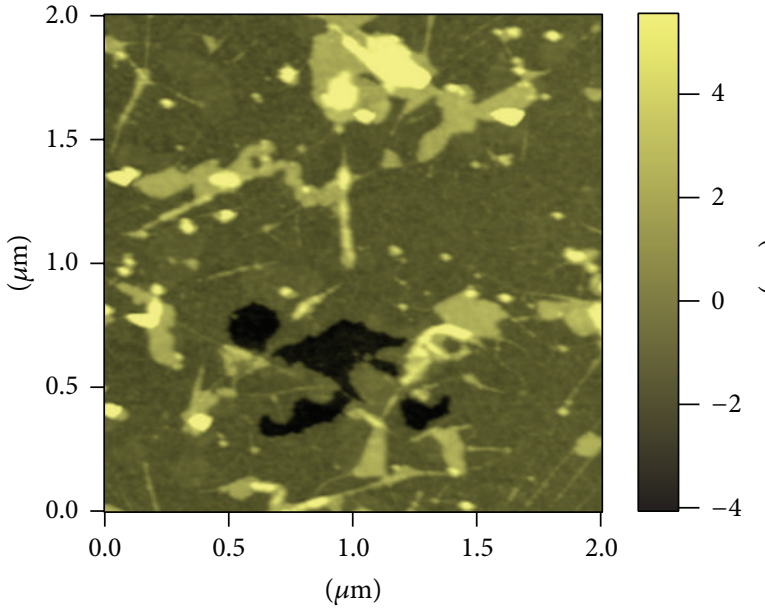

(a)

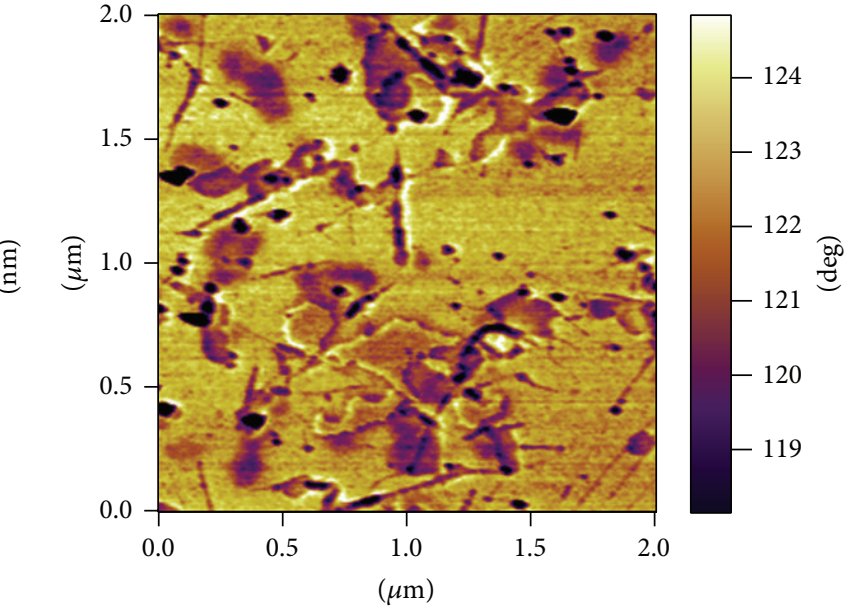

(b)

FIGURE 12: EFM analysis SWCNTs dispersed in water by DOC $(10 \mathrm{mg} / \mathrm{mL})$. (a) AFM image and (b) its corresponding Nap phase are shown. In the height tracing, nanotubes are wrapped by the surfactants where the Nap phase shows the presence of surfactants by phase difference. Presence of surfactants reduces phase as it changes surface properties of the CNTs.

On the other hand, organic solvents can dissolve only a low amount of SWCNTs but still better to be used in organic solar cells owing to that they offer high pure SWCNTs. The resonication of the centrifuged solution plays a crucial role towards obtaining a homogeneous dispersion of SWCNTs.

\section{Conflict of Interests}

The authors declare that there is no conflict of interests regarding the publication of this paper.

\section{Acknowledgments}

The authors would like to acknowledge the valuable support from Masdar Institute and contributions from Dr. Matteo Chiesa and the people from his group (Laboratory of Energy and Nanoscience).

\section{References}

[1] E. Kymakis, I. Alexandrou, and G. A. J. Amaratunga, "High open-circuit voltage photovoltaic devices from carbonnanotube-polymer composites," Journal of Applied Physics, vol. 93, no. 3, pp. 1764-1768, 2003.

[2] L. Hu, D. S. Hecht, and G. Grüner, "Carbon nanotube thin films: fabrication, properties, and applications," Chemical Reviews, vol. 110, no. 10, pp. 5790-5844, 2010.

[3] R. H. Baughman, A. A. Zakhidov, and W. A. De Heer, "Carbon nanotubes-The route toward applications," Science, vol. 297, no. 5582, pp. 787-792, 2002.

[4] G. Christensen, H. Younes, H. Hong, and G. P. Peterson, "Alignment of carbon nanotubes comprising magnetically sensitive metal oxides by different chemical surfactants," Journal of Nanofluids, vol. 2, pp. 25-28, 2013.

[5] S. Neupane, S. Khatiwad, C. Jaye et al., "Single-walled carbon nanotubes coated by $\mathrm{Fe} 2 \mathrm{O} 3$ nanoparticles with enhanced 
magnetic properties," ECS Journal of Solid State Science and Technology, vol. 3, no. 8, pp. M39-M44, 2014.

[6] H. Liu, H. Ji, H. Hong, and H. Younes, “Tribological properties of carbon nanotube grease," Industrial Lubrication and Tribology, vol. 66, no. 5, pp. 579-583, 2014.

[7] J. Mao, Q. Liu, X. Lv et al., "A water-soluble hybrid material of single-walled carbon nanotubes with an amphiphilic poly(phenyleneethynylene): preparation, characterization, and photovoltaic properties," Journal of Nanoscience and Nanotechnology, vol. 7, no. 8, pp. 2709-2718, 2007.

[8] J. Arranz-Andrés and W. J. Blau, "Enhanced device performance using different carbon nanotube types in polymer photovoltaic devices," Carbon, vol. 46, no. 15, pp. 2067-2075, 2008.

[9] K. M. Coakley and M. D. McGehee, "Conjugated polymer photovoltaic cells," Chemistry of Materials, vol. 16, no. 23, pp. 4533-4542, 2004.

[10] L. Liu and G. Li, "Electrical characterization of single-walled carbon nanotubes in organic solar cells by Kelvin probe force microscopy," Applied Physics Letters, vol. 96, no. 8, Article ID 083302, 2010.

[11] S. Reich, C. Thomsen, and P. Ordejón, "Electronic band structure of isolated and bundled carbon nanotubes," Physical Review B-Condensed Matter and Materials Physics, vol. 65, no. 15, Article ID 155411, 2002.

[12] L. Jiang, L. Gao, and J. Sun, "Production of aqueous colloidal dispersions of carbon nanotubes," Journal of Colloid and Interface Science, vol. 260, no. 1, pp. 89-94, 2003.

[13] Y. Tan and D. E. Resasco, "Dispersion of single-walled carbon nanotubes of narrow diameter distribution," Journal of Physical Chemistry B, vol. 109, no. 30, pp. 14454-14460, 2005.

[14] R. Rastogi, R. Kaushal, S. K. Tripathi, A. L. Sharma, I. Kaur, and L. M. Bharadwaj, "Comparative study of carbon nanotube dispersion using surfactants," Journal of Colloid and Interface Science, vol. 328, no. 2, pp. 421-428, 2008.

[15] A. J. Blanch, C. E. Lenehan, and J. S. Quinton, "Optimizing surfactant concentrations for dispersion of single-walled carbon nanotubes in aqueous solution," Journal of Physical Chemistry B, vol. 114, no. 30, pp. 9805-9811, 2010.

[16] V. C. Moore, M. S. Strano, E. H. Haroz et al., "Individually suspended single-walled carbon nanotubes in various surfactants," Nano Letters, vol. 3, no. 10, pp. 1379-1382, 2003.

[17] M. F. Islam, E. Rojas, D. M. Bergey, A. T. Johnson, and A. G. Yodh, "High weight fraction surfactant solubilization of singlewall carbon nanotubes in water," Nano Letters, vol. 3, no. 2, pp. 269-273, 2003.

[18] N. Grossiord, P. van der SchooT, J. Meuldijk, and C. E. Koning, "Determination of the surface coverage of exfoliated carbon nanotubes by surfactant molecules in aqueous solution," Langmuir, vol. 23, no. 7, pp. 3646-3653, 2007.

[19] H. Wang, "Dispersing carbon nanotubes using surfactants," Current Opinion in Colloid and Interface Science, vol. 14, no. 5, pp. 364-371, 2009.

[20] P. Angelikopoulos, A. Gromov, A. Leen, O. Nerushev, H. Bock, and E. E. B. Campbell, "Dispersing individual single-wall carbon nanotubes in aqueous surfactant solutions below the cmc," Journal of Physical Chemistry C, vol. 114, no. 1, pp. 2-9, 2010.

[21] M. S. Strano, V. C. Moore, M. K. Miller et al., "The role of surfactant adsorption during ultrasonication in the dispersion of single-walled carbon nanotubes," Journal of Nanoscience and Nanotechnology, vol. 3, no. 1-2, pp. 81-86, 2003.
[22] B. Ratier, J.-M. Nunzi, M. Aldissi, T. M. Kraft, and E. Buncel, "Organic solar cell materials and active layer designsimprovements with carbon nanotubes: a review," Polymer International, vol. 61, no. 3, pp. 342-354, 2012.

[23] C.-K. Chang, J.-Y. Hwang, W.-J. Lai et al., "Influence of solvent on the dispersion of single-walled carbon nanotubes in polymer matrix and the photovoltaic performance," Journal of Physical Chemistry C, vol. 114, no. 24, pp. 10932-10936, 2010.

[24] S. Manivannan, I. O. Jeong, J. H. Ryu et al., "Dispersion of single-walled carbon nanotubes in aqueous and organic solvents through a polymer wrapping functionalization," Journal of Materials Science: Materials in Electronics, vol. 20, no. 3, pp. 223-229, 2009.

[25] Q. Cheng, S. Debnath, L. O’Neill, T. G. Hedderman, E. Gregan, and H. J. Byrne, "Systematic study of the dispersion of swnts in organic solvents," Journal of Physical Chemistry C, vol. 114, no. 11, pp. 4857-4863, 2010.

[26] K. D. Ausman, R. Piner, O. Lourie, R. S. Ruoff, and M. Korobov, "Organic solvent dispersions of single-walled carbon nanotubes: Toward solutions of pristine nanotubes," Journal of Physical Chemistry B, vol. 104, no. 38, pp. 8911-8915, 2000.

[27] K. K. Kim, D. J. Bae, C.-M. Yang, K. H. An, J. Y. Lee, and Y. H. Lee, "Nanodispersion of single-walled carbon nanotubes using dichloroethane," Journal of Nanoscience and Nanotechnology, vol. 5, no. 7, pp. 1055-1059, 2005.

[28] H. Younes, G. Christensen, M. Liu, H. Hong, Q. Yan, and T. Lin, "Alignment of carbon nanofibers in water and epoxy by external magnetic field," Journal of Nanofluids, vol. 2, no. 1, pp. 25-28, 2014.

[29] H. Younes, G. Christensen, X. Luan, H. Hong, and P. Smith, "Effects of alignment, $\mathrm{pH}$, surfactant, and solvent on heat transfer nanofluids containing $\mathrm{Fe}_{2} \mathrm{O}_{3}$ and $\mathrm{CuO}$ nanoparticles," Journal of Applied Physics, vol. 111, no. 6, Article ID 064308, 2012.

[30] H. Younes, G. Christensen, H. Hong, and G. P. Peterson, "Alignment of different functional single wall carbon nanotubes using $\mathrm{Fe}_{2} \mathrm{O}_{3}$ nanoparticles under external magnetic field," Journal of Nanofluids, vol. 2, no. 1, pp. 4-10, 2013.

[31] A. V. Naumov, S. Ghosh, D. A. Tsyboulski, S. M. Bachilo, and R. B. Weisman, "Analyzing absorption backgrounds in singlewalled carbon nanotube spectra," ACS Nano, vol. 5, no. 3, pp. 1639-1648, 2011. 

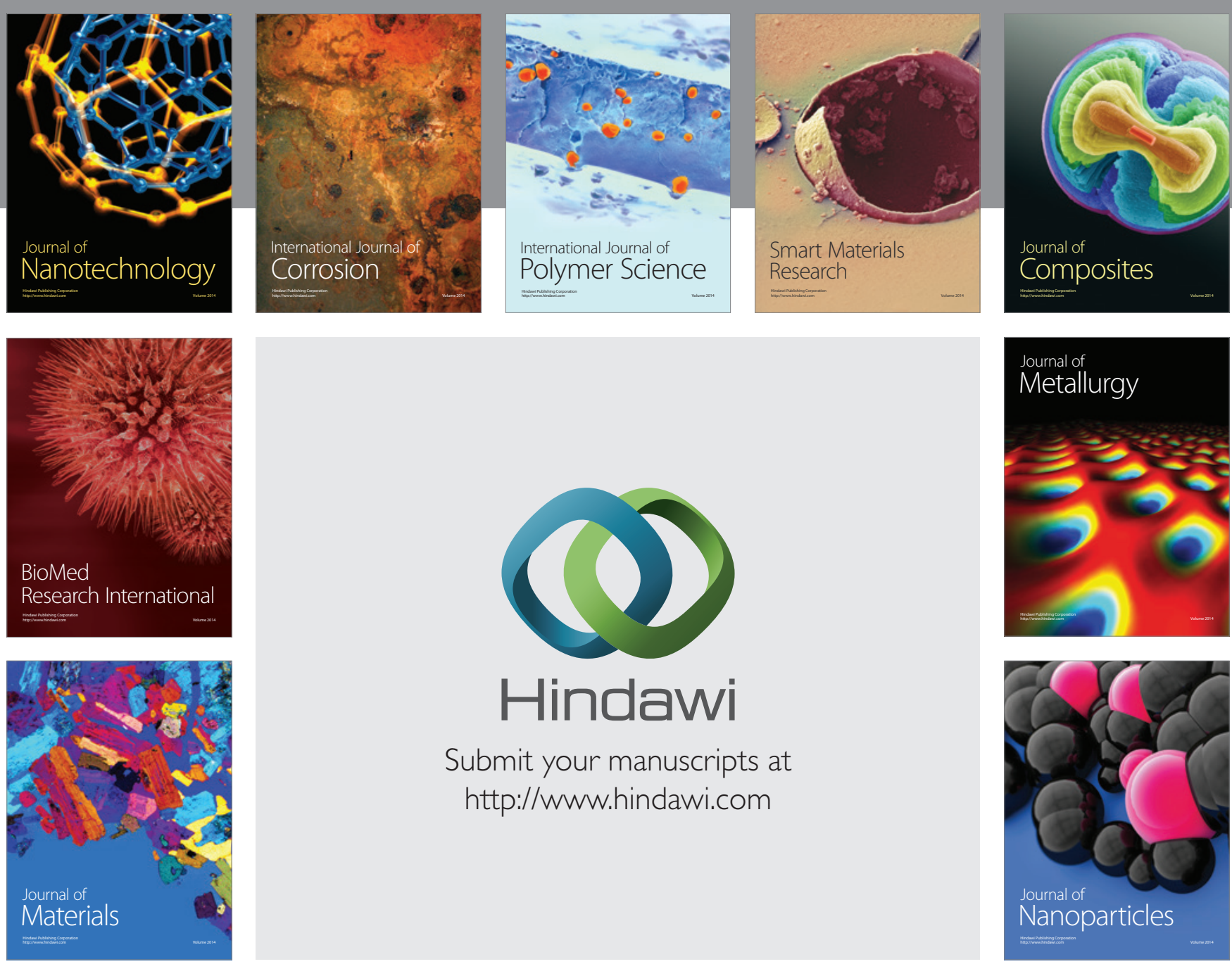

Submit your manuscripts at http://www.hindawi.com
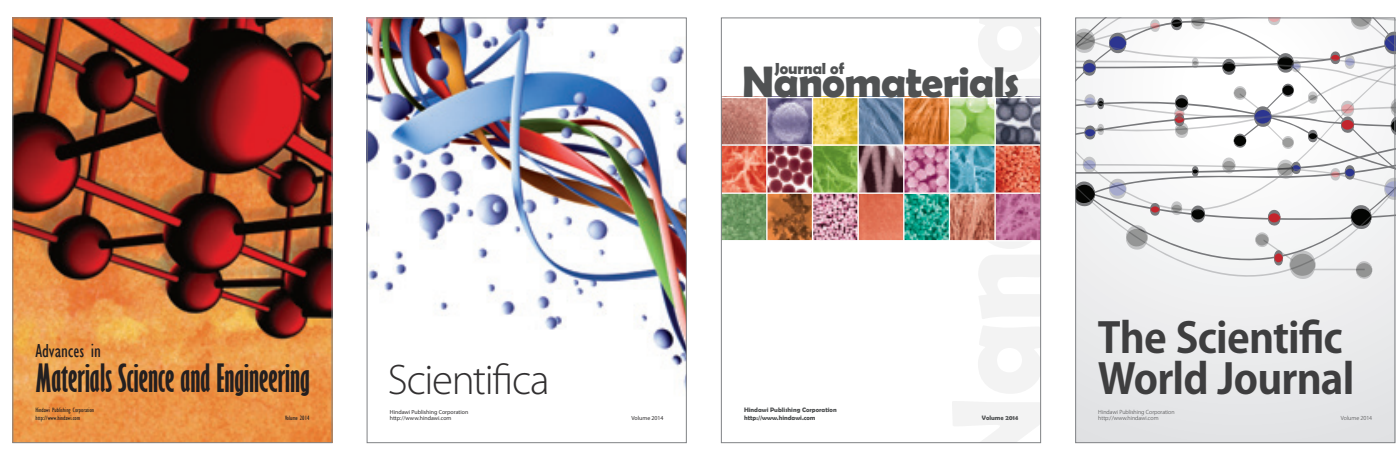

\section{The Scientific World Journal}
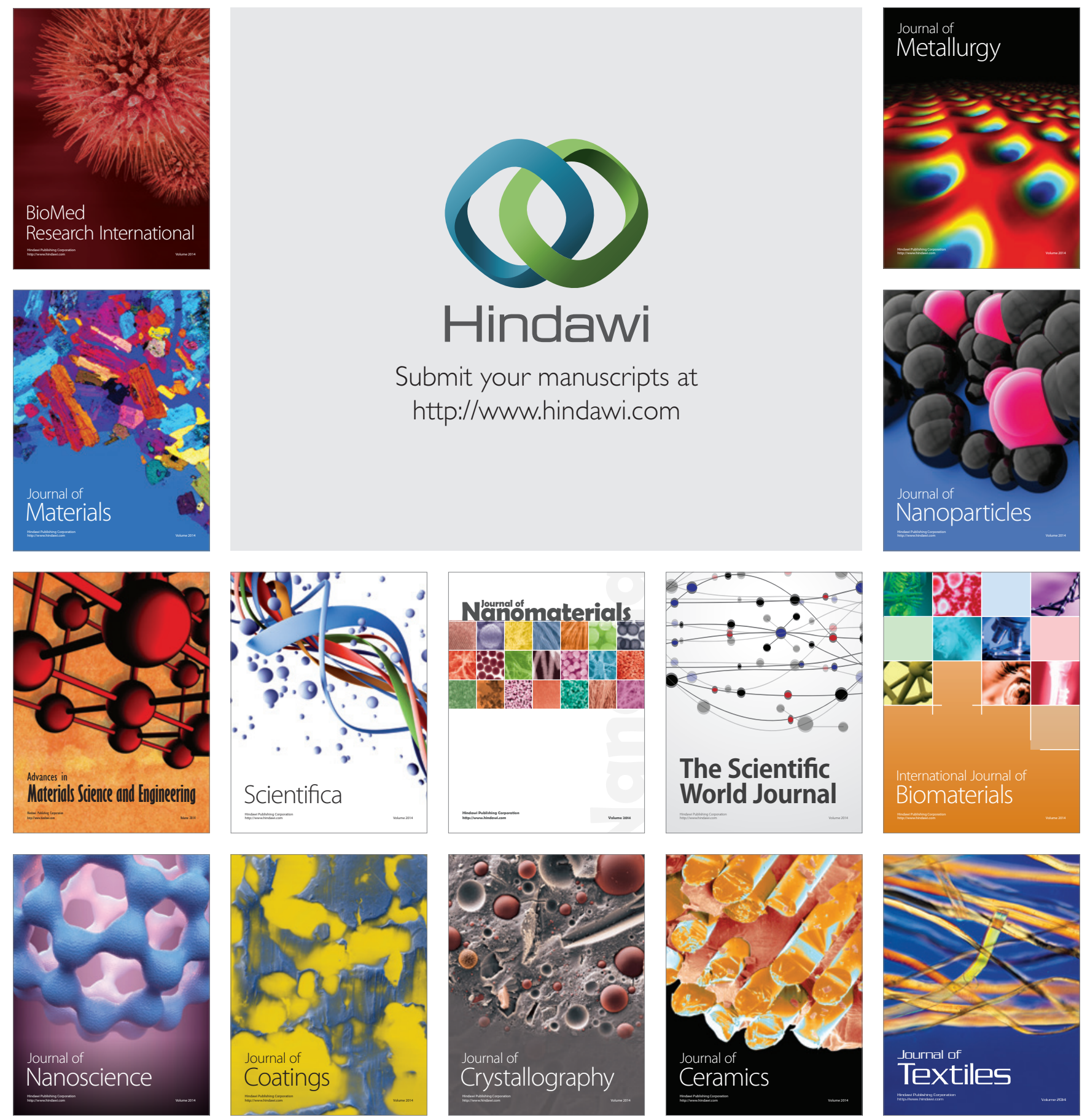